\title{
Evaluation of the impact of treatment delays and dose reductions in human ovarian cancer orthotopic mouse models
}

\author{
Scott Mosley ${ }^{1 \#}$, Jing-Hong Chen ${ }^{2 \#}$, Anjali Gaikwad ${ }^{3}$, Elizabeth K. Nugent ${ }^{3}$ and Judith A. Smith ${ }^{3 *}$ \\ ${ }^{1}$ Department of Pharmacotherapy and Translational Research, University of Florida College of Pharmacy, Gainsville, FL, USA \\ ${ }^{2}$ Shanghai Jiao Tong University School of Medicine, Shanghai, China \\ ${ }^{3}$ Department of Obstetrics, Gynecology and Reproductive Sciences, University of Texas Health Science Center Medical School at Houston; Houston, TX, USA \\ \#Both of them contributed equally
}

\begin{abstract}
The primary objective of this study was to quantify the impact of treatment delay and dose reductions in an in vivo orthotopic ovarian cancer model. In addition, this study evaluated the role of growth factors in achieving "full dose on time". HeyA-8 and SKOV3.ip1ovarian cancer orthotopic mouse models were used determine the effect of paclitaxel/carboplatin treatment delays or dose reductions on tumor response and overall survival. The addition of growth factor support with pegfilgrastim and darbepoetin was also evaluated in treatment control arm. The results indicated that full dose of paclitaxel (20 mg/kg) and carboplatin (50 mg/ $\mathrm{kg}) \mathrm{delivered} \mathrm{on}$ time (every 21 days) achieved better tumor response in both aggressive (HeyA-8) and metastatic (SKOV3.ip1) human ovarian tumors compared to treatment delay or dose reduction arms. In the HeyA-8 orthotopic model treatment delay $(\mathrm{P}<0.05)$ resulted in a significant reduction in overall survival compared to on time, full dose treatment control arm and the dose reduction decreased overall survival but was not statistically significant. However, in the SKOV3.ip1orthotopic model a treatment delay $(\mathrm{P}<0.01)$ or dose reduction $(\mathrm{P}<0.05)$ both resulted in a significant reduction in overall survival compared to on time, full dose treatment control arm. In both orthotopic models, the addition of pegfilgrastim $(1000 \mu \mathrm{g} / \mathrm{kg})$ and darbepoetin $(10 \mu \mathrm{g} / \mathrm{kg})$ delayed the onset of ascites formation and was associated with an improved overall survival rate. Treatment delays and dose reductions had a negative impact on tumor response and overall survival compare with on-time, full dose treatment controls. In addition, use of growth factor agents improved overall survival and tumor responses.
\end{abstract}

\section{Introduction}

Ovarian cancer is the most lethal gynecological cancer and the fifth most common cause of malignancy in women [1]. Ovarian cancer is difficult to detect and diagnose early, therefore patients often initially present with advanced disease and poor prognosis. The current standard of care includes primary tumor debulking surgery followed by cytotoxic chemotherapy with $70 \%$ of patients achieving a complete initial clinical response. The current standard of care for primary chemotherapy is six cycles of combination of taxanes agent plus a platinum agent given once every three weeks [2]. Although multiple studies have evaluated different dosing parameters and routes of administration to reduce toxicity and optimize efficacy, often clinical practice requires dose reductions or treatment delays to complete six cycles of therapy [3,4].

It is well established that granulocyte-colony stimulating factor (G-CSF) minimizes the magnitude and duration of neutropenia and improves overall treatment outcomes primarily by decreasing the risk of infection, hospitalization, and potentially fatal complications from sepsis [5-9]. In addition to increasing the risk of developing febrile neutropenia, prolonged neutropenia often leads to delays in treatment and dose reductions of planned chemotherapy $[7,8]$. Anemia is another common development during chemotherapy that is responsible for dose reductions and treatment delays; and prevention of anemia has been suggested to improve treatment outcomes both efficacy and quality of life. Intratumoral hypoxia is associated with promoting resistance to chemotherapy and/or radiotherapy, genetic instability, angiogenesis, and inhibition of apoptosis [9-12]. New and improved growth factor support with agents such as pegfilgrastim and darbepoetin alfa have the potential to improve the management of chemotherapy-induced neutropenia and anemia, respectively [13].

There is minimal information about the impact of treatment delays or dose reductions on treatment responses and overall outcomes in cancer patients. The primary objective of this study was to quantify the impact of dose reductions and treatment delays in an in vivo orthotopic ovarian cancer model. In addition, the role of growth factors to improve the ability of patients to receive "full dose on time" was evaluated.

\section{Materials and methods}

\section{Cell culture}

HeyA-8 human ovarian cancer cells obtained from American Type Culture Collection (Rockville, MD) were cultured in RPMI 1640 supplemented (GIBCO) with 10\% fetal bovine serum (Invitrogen), SKOV3.ip1 human ovarian cancer cells were generously provided by

Correspondence to: Judith A. Smith, Pharm.D., BCOP, CPHQ, FCCP, FISOPP, Department of Obstetrics, Gynecology and Reproductive Sciences, The University of Texas Health Sciences Center, 6431 Fannin Street, Rm 3.152, Houston, TX 77030, USA, Office: 1-713-500-6408; Fax: 1-713-500-5474; E-mail: Judith.Ann.Smith@uth.tmc.edu

Key words: dose reductions, treatment delays, chemotherapy, ovarian cancer

Received: October 10, 2015; Accepted: October 24, 2015; Published: October 27, 2015 
Dr. Josh Fidler, The University of Texas, MD Anderson Cancer Center (UTMDACC) were cultured in Eagle's Minimum Essential Medium (MEM) with Earle's basic salt solution (BSS) and non-essential amino acids (NEAA), $1 \mathrm{mmol} / \mathrm{L}$ sodium pyruvate, $2 \mathrm{X}$ MEM vitamin solution with $10 \%$ fetal bovine serum and $1.5 \mathrm{mmol} / \mathrm{L} \mathrm{L}$-glutamine. Cell lines used for this study were less than 10 passages to ensure the platinum resistance or sensitivity, as well as preventing major changes in cell line characteristics.

\section{Drugs}

Paclitaxel $6 \mathrm{mg} / \mathrm{mL}$ solution (Eli Lilly, Inc Indianapolis, IN) and carboplatin $10 \mathrm{mg} / \mathrm{mL}$ solution (Eli Lilly, Inc Indianapolis, IN) were purchased from the UTMDACC pharmacy department. All drugs were diluted in fresh $0.9 \%$ sodium chloride immediately before each drug administration. Growth factor agents including pegfilgrastim $10 \mathrm{mg} /$ $\mathrm{mL}$ and darbepoetin $10 \mathrm{mg} / \mathrm{mL}$ were generously provided from Amgen Oncology Company (Thousand. Oaks, CA).

\section{HeyA-8 and SKOV3.iplovarian cancer orthotopic mouse models}

The protocol was reviewed and approved by the institutional animal care and utilization committee (IACUC) prior to initiating any animal work. All mice were handled according to the Guide for the Care and Use of Laboratory Animals. HeyA-8 or SKOV3.ip1 cells were grown to confluence and harvested by trypsinization with $0.25 \mathrm{mg} / \mathrm{mL}$ trypsin/
EDTA and suspended in PBS before inoculation into mice. HeyA-8 cells $\left(3 \times 10^{6}\right)$ and SKOV3.ip1 cells $\left(9 \times 10^{6}\right)$ were collected, counted and dispersed in a volume of $200 \mu \mathrm{L}$ of PBS or serum-free cell culture medium and were injected into the peritoneal cavities of six-week-old female nude mice

Two intraperitoneal (IP) ovarian cancer orthotopic models were utilized including the HeyA- 8 orthotopic model to represent chemosenstive, aggressive ovarian cancer or SKOV3.ip1 to represent multi-drug resistant metastatic ovarian cancer, to evaluate the dose and timing of administration of taxane/platinum combination regimen. There were a total of six groups including: untreated, vehicle alone, treatment control A paclitaxel/carboplatin alone once every three weeks, and treatment control B paclitaxel/carboplatin + GCSF once every three weeks, experimental treatment delay group and experimental dose reduction group for each orthotopic model with ten mice in each group (Figure 1). A total of 90 mice per each cell line and 180 mice were required to complete the study.

\section{Treatment}

Ten days after tumor inoculation, mice were randomized to receive administration of IV injection of normal saline, or paclitaxel $20 \mathrm{mg} /$ $\mathrm{kg} \mathrm{IV} \mathrm{and} \mathrm{carboplatin} 50 \mathrm{mg} / \mathrm{kg}$ IV alone, or paclitaxel $20 \mathrm{mg} / \mathrm{kg}$ IV and carboplatin $50 \mathrm{mg} / \mathrm{kg}$ IV on day one then pegfilgrastim $1000 \mu \mathrm{g} /$ $\mathrm{kg}$ subcutaneous (subQ) and darbepoetin $10 \mu \mathrm{g} / \mathrm{kg}$, subQ on day two. Doses selected for this study were based on previous animal studies and

Untreated Control Mice (10 mice) - No treatment

Vehicle alone Control Mice (10 mice) - No treatment, just diluents

TX alone Control Mice (10 mice) - ON TIME, FULL DOSE

Paclitaxel $(20 \mathrm{mg} / \mathrm{kg})+$ Carboplatin $(50 \mathrm{mg} / \mathrm{kg})$ each will be given as single $0.15 \mathrm{~mL} \mathrm{IV}$

GF support + TX Control Mice (10 mice) - ON TIME, FULL DOSE + growth factor

support

**Please note all IV injections done with a 23-gauge needle

Day one: Paclitaxel $(20 \mathrm{mg} / \mathrm{kg})+$ Carboplatin $(50 \mathrm{mg} / \mathrm{kg})$ each will be given as single $0.15 \mathrm{~mL}$

\section{Experiment One \\ Treatment Delay \\ (10 mice per group)}

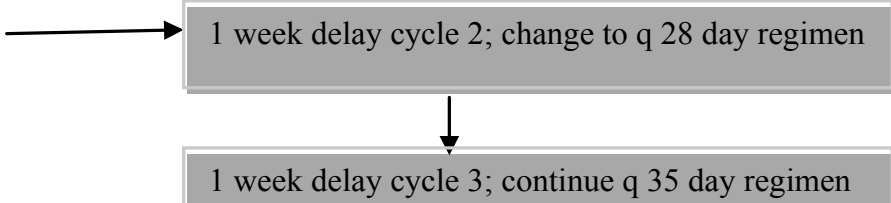

1 week delay cycle 3 ; continue q 35 day regimen

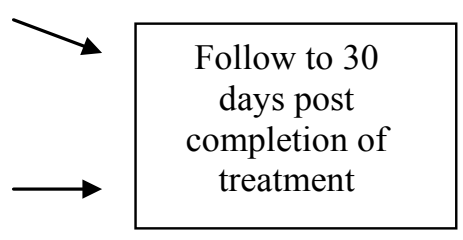

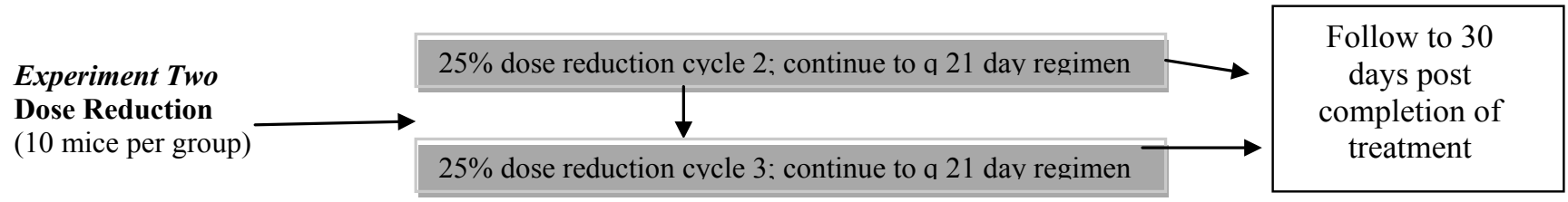

Figure 1. Study Treatment flow chart. 90 mice per experiment two cell lines $=* 180$ mice. 
are comparable to respective clinical doses tolerated in humans [14].

Each treatment group initiated chemotherapy with paclitaxel 20 $\mathrm{mg} / \mathrm{kg}$ IV and carboplatin $50 \mathrm{mg} / \mathrm{kg}$ IV on day one of the each 21 -day cycle with a total of three planned cycles. The control treatment groups continued to receive full dose paclitaxel $20 \mathrm{mg} / \mathrm{kg}$ IV and carboplatin $50 \mathrm{mg} / \mathrm{kg} \mathrm{IV}$ on day one followed by pegfilgrastim $1000 \mu \mathrm{g} / \mathrm{kg}$ subQ + darbepoetin $10 \mu \mathrm{g} / \mathrm{kg}$ subQ on day two, every 21 days, for three cycles. In the treatment delay experiment arm, there were empiric treatment delays in two treatment delay groups occurring on either cycle 2 or cycle 3 , respectively (Figure 1). In the dose reduction experimental arm there were empiric dose reductions of $25 \%$ in two of the dose reduction groups occurring on either cycle 2 or cycle 3 , respectively (Figure 1).

\section{Tumor response determination}

As surrogates to reflect both increasing ascites accumulation and tumor burden the body weight and abdominal circumference were quantified three times a week as average measurement for each parameter. Abdominal circumference measurements were obtained with a special device (restraining tube with fixed soft ruler) at the body's widest mid-point marked and monitored throughout the experimental period while the animal was held in a prone position. In addition, mice were monitored three times a week for evidence of signs/symptoms of moriboundity (weight loss, anorexia, hunching, listlessness, extensively enlarged abdominal cavity, lethargy, etc.). Paracentesis was performed to remove fluid up to $1 \mathrm{~mL}$ as appropriate to relieve abdominal bloating and fluid volume was measured up to three times for each animal before sacrificing. At the end of the experiment, all remaining mice were sacrificed humanely via $\mathrm{CO}_{2}$ inhalation followed by cervical dislocation.

\section{Data analysis}

Descriptive statistics were completed to evaluate delay in tumor growth and/or tumor regression and contrasted with respective to toxicity profiles. T-test analysis was used in the data analysis to evaluate and compare the activity and toxicity, respectively, for each experimental arm compared to the treatment control arms with and without growth factors.

\section{Results}

A summary of the impact of dose reductions and treatment delays on the overall survival rate of the HeyA-8 and SKOV3.ip1 tumor bearing mice is reported in Figure 2 and Table 1.

In the HeyA-8 non-treatment and vehicle control group, all mice died before day 40 . The mean survival time of the HeyA- 8 vehicle control group was $36.5 \pm 2$ days after tumor implantation. In the HeyA-8 paclitaxel/carboplatin treatment control group, however, two animal deaths occurred during the study and the remaining animals were still alive on day 40 . The mean overall survival time in the HeyA-8 paclitaxel/carboplatin treatment group was $44.5 \pm 4.9$ days (Figure 2a).

In the HeyA-8 treatment delay group, four mice died during the study. The mean overall survival was $30.5 \pm 4.2$ days which was significantly reduced compared to the treatment control groups $(\mathrm{P}<0.05)$ (Table 1). For the HeyA-8 dose reduction group, the mean overall survival was $44 \pm 3.8$ days which was not statistically significant compared to the HeyA-8 full dose treatment control group.

Among the treatment groups, paclitaxel/carboplatin followed by growth factor (GF) agents had the longest median survival and the most surviving ( 65 days, 1 surviving mouse). In the HeyA-8 paclitaxel/ carboplatin $+\mathrm{GF}$ control group, the two animal deaths occurred during the study and the remaining animals were still alive after day 50 . The mean overall survival time was $55 \pm 5.8$ days which was statistically significant compared to the HeyA- 8 untreated controls $(\mathrm{P}<0.01)$ (Figure 1a). Paclitaxel/carboplatin +GF was improved but did not statistically differ compared to full dose, on time treatment control group ( $\mathrm{p}=0.09$ ). However, Paclitaxel/carboplatin $+\mathrm{GF}$ was superior to both the treatment delay $(\mathrm{p}<0.001)$ and dose reduction group $(\mathrm{p}=0.06)$.

Similar findings were observed in the multi-drug resistant SKOV3. ip1 orthotopic model study. In SKOV3.ip1 arm nine of the non-
A

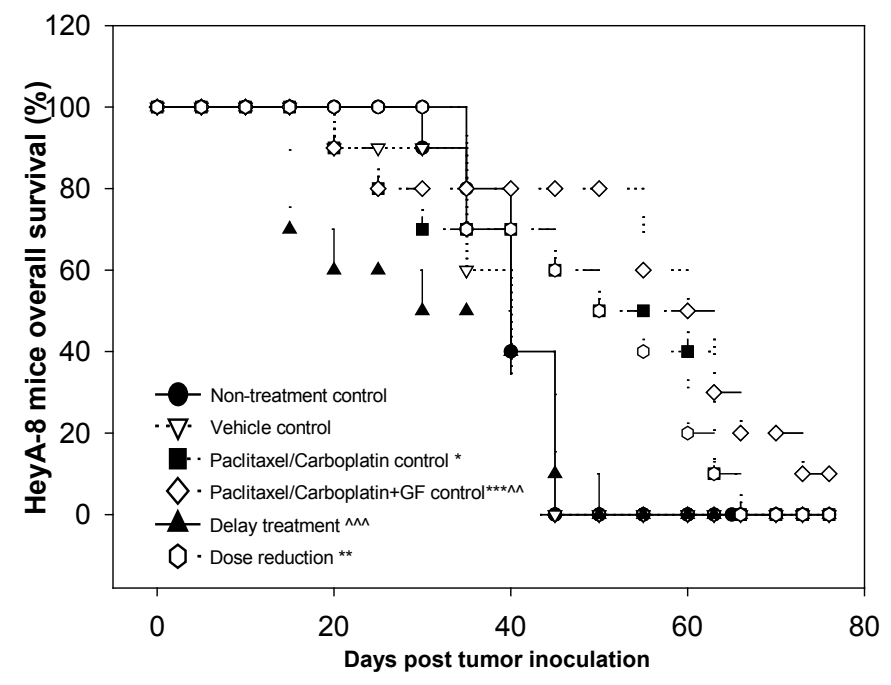

B

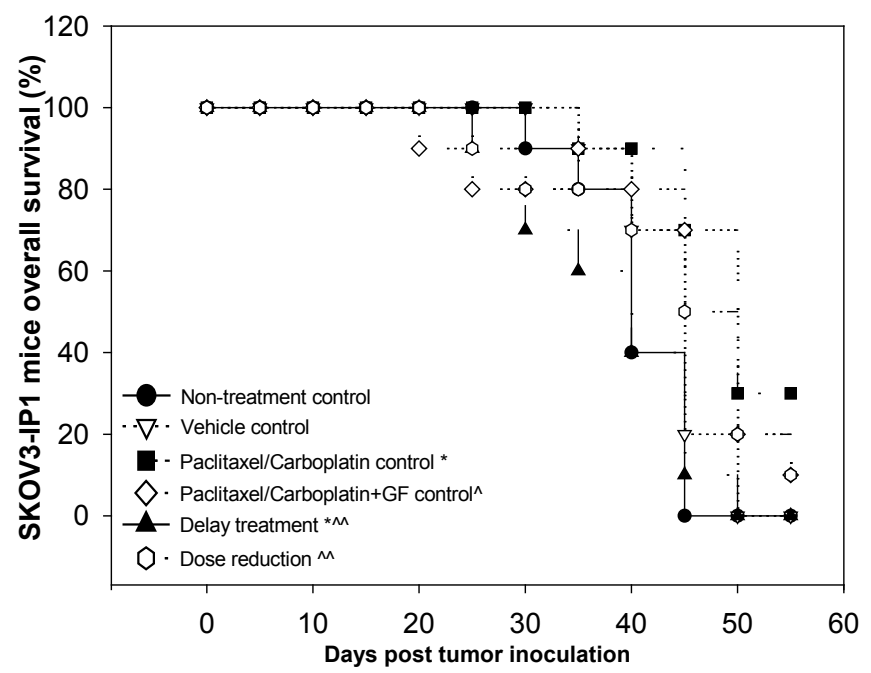

Figure 2. Summary figures of the overall survival. The survival curves of the six groups illustrating the survival of mice non-treated and different treatment arms for the HeyA-8 ovarian cancer orthotopic model (2A) and SKOV3.iplovarian cancer orthotopic model (2B). Survival rate of paclitaxel ( $20 \mathrm{mg} / \mathrm{kg})+$ carboplatin $(50 \mathrm{mg} / \mathrm{kg})+/$ - growth factor support groups showed a significant improvement in the survival rate. 
Table 1. Overall Survival and living status change summary for HeyA-8 and SKOV3.ip1mice.

\begin{tabular}{|c|c|c|c|}
\hline Cell type & Treatment Group & Ave Survived days $\pm \mathrm{SE}$ & Bodyweight change $\pm \mathrm{SE}$ \\
\hline \multirow[t]{6}{*}{ HeyA-8 } & Non-treatment control & $37.5 \pm 1.1$ & $5.5 \pm 0.6$ \\
\hline & Vehicle control & $36.5 \pm 2$ & $4.2 \pm 0.9$ \\
\hline & Paclitaxel/Carboplatin treatment control & $44.5 \pm 4.9$ & $2.4 \pm 0.5^{* * * \#}$ \\
\hline & Paclitaxel/Carboplatin +GF control & $55 \pm 5.8^{* * \#}$ & $1.6 \pm 0.3 * * * \#$ \\
\hline & Treatment delay (H1A) & $30.5 \pm 4.2^{\wedge}$ & $2.6 \pm 0.5^{* *}$ \\
\hline & Dose reduction $(\mathrm{H} 2 \mathrm{~A})$ & $44 \pm 3.8$ & $1.3 \pm 0.3 * * * \#$ \\
\hline \multirow[t]{6}{*}{ SKOV3-IP1 } & Non-treatment control & $38.5 \pm 0.8$ & $4.5 \pm 0.6$ \\
\hline & Vehicle control & $38 \pm 1.3$ & $4.6 \pm 0.7$ \\
\hline & Paclitaxel/Carboplatin treatment control & $46.5 \pm 1.8^{* * * \ldots \# \#}$ & $2.9 \pm 0.9$ \\
\hline & Paclitaxel/Carboplatin +GF control & $44.5 \pm 3.2 * \#$ & $1.7 \pm 0.7 * * \#$ \\
\hline & Treatment delay (S1A) & $38 \pm 2.4^{\wedge} \wedge$ & $4 \pm 1$ \\
\hline & Dose reduction (S2A) & $39 \pm 2.8^{\wedge}$ & $4.2 \pm 0.5$ \\
\hline
\end{tabular}

Note: Symbol * compared to non-treatment control group. Symbol ${ }^{\#}$ compared to vehicle control group.

Symbol ${ }^{\wedge}$ compared to paclitaxel/carboplatin treatment control group. ${ }^{*}$ For statistically significance, one symbol indicated $\mathrm{P}<0.05$, two symbols indicated $\mathrm{P}<0.01$ and three symbols indicated $\mathrm{P}<0.001$

Table 2: Ascites formation analysis table for HeyA-8 and SKOV3.ip1mice.

\begin{tabular}{|c|c|c|c|c|}
\hline Cell type & Treatment & Ave days onset of ascites $\pm \mathrm{SE}$ & Ave ascites volume $\pm \mathrm{SE}$ & Ave waistline changes $\pm \mathrm{SE}$ \\
\hline \multirow[t]{6}{*}{ HeyA-8 } & Non-treatment control & $32.9 \pm 0.8$ & $0.9 \pm 0.2$ & $1.5 \pm 0.2$ \\
\hline & Vehicle control group & $33.1 \pm 0.8$ & $1.1 \pm 0.4$ & $1.3 \pm 0.2$ \\
\hline & Paclitaxel/Carboplatin treatment control & $42.5 \pm 5.6$ & $0.45 \pm 0.02 *$ & $0.4 \pm 0.2 * * * \ldots \#$ \\
\hline & Paclitaxel/Carboplatin + GF control & $53.1 \pm 5 * * \#$ & $0.7 \pm 0.2$ & $0.3 \pm 0.1 * * * \ldots \#$ \\
\hline & Treatment delay (H1A) & $36 \pm 2.3$ & $0.8 \pm 0.1$ & $1 \pm 0.2^{\wedge}$ \\
\hline & Dose reduction $(\mathrm{H} 2 \mathrm{~A})$ & $31.3 \pm 1.5$ & $0.7 \pm 0.1$ & $0.5 \pm 0.2^{*}$ \\
\hline \multirow[t]{6}{*}{ SKOV3.ip1 } & Non-treatment control & $31.5 \pm 1.5$ & $0.8 \pm 0.1$ & $1.6 \pm 0.1$ \\
\hline & Vehicle control group & $31.7 \pm 1.1$ & $0.7 \pm 0.2$ & $1.3 \pm 0.2$ \\
\hline & Paclitaxel/Carboplatin treatment control & $40 \pm 1.9^{*}$ & $0.4 \pm 0.1$ & $0.4 \pm 0.1 * * * \ldots \#$ \\
\hline & Paclitaxel/Carboplatin treatment + GF control & $40 \pm 2 *$ & $0.5 \pm 0.04$ \# & $0.4 \pm 0.1 * * * \# \#$ \\
\hline & Treatment delay (S1A) & $33.3 \pm 0.8$ & $1.1 \pm 0.4^{\wedge}$ & $1.3 \pm 0.2^{\wedge}$ \\
\hline & Dose Reduction (S2A) & $37 \pm 2.1$ & $0.7 \pm 0.1$ & $1.1 \pm 0.3^{\wedge}$ \\
\hline
\end{tabular}

Note: Symbol * compared to non-treatment control group. Symbol ${ }^{*}$ compared to vehicle control group. Symbol^ compared to paclitaxel/carboplatin treatment control group. For statistical significance, one symbol $\wedge$ indicated $\mathrm{P}<0.05$, two symbols $\wedge \wedge$ indicated $\mathrm{P}<0.01$ and three symbols $\wedge \wedge \wedge$ indicated $\mathrm{P}<0.001$.

treatment or vehicle control mice died before the day 40 and only one mouse survived until day 45 . The mean overall survival time of the SKOV3.ip1vehicle group was $38 \pm 1.33$ days after tumor inoculation. In the SKOV3.iplfull dose, on time paclitaxel/carboplatin treatment control group, seven animal deaths prior to day 50 post inoculation. The mean overall survival time in the SKOV3.ip1treatment control group was $46.5 \pm 1.8$ days $(\mathrm{P}<0.001)$. In the SKOV3.ip1paclitaxel/carboplatin $+\mathrm{GF}$ group there were no deaths prior to day 55 post inoculation. The mean overall survival for the SKOV3.iplpaclitaxel/carboplatin +GF group was $44.5 \pm 3.2$ days which was not statistically different than the SKOV3.ip1full dose, on time paclitaxel/carboplatin treatment control group (Figure 2b).

In the SKOV3.ip1treatment delay group all mice died prior to day 45 of the study. The mean overall survival was $38.0 \pm 2.4$ days which was significantly reduced compared to the full dose, on time paclitaxel/ carboplatin treatment control group $(\mathrm{p}<0.01)$. The SKOV3.ip1dose reduction group had a mean overall survival of $39 \pm 2.8$ days which was also significantly reduced compared to the full dose, on time paclitaxel/ carboplatin treatment control group $(\mathrm{P}<0.05)$.

\section{Evaluation of ascites formation}

At postmortem examination, tumors were found on the surface of the peritoneum, uterus on the diaphragm, in the liver, and on the intestines in both control and treated groups. Ascites volumes were quantified during each experiment and postmortem examination. Groups were compared and the results are depicted in Table 2. The ascites onset in the HeyA-8 non-treatment and vehicle control groups was very early with the mean onset of ascites formation of $2.9 \pm 0.8$ and $33.1 \pm 0.8$ days, respectively. However, in the HeyA-8 paclitaxel/ carboplatin + GF control group, a significant delay in the ascites formation was observed with a mean onset time of $53.1 \pm 5$ days 
$(\mathrm{P}<0.01)$. A similar improvement was observed in the SKOV3.ip1 paclitaxel/carboplatin + GF control group having delayed onset of ascites formation compared with non-treatment groups $(\mathrm{p}<0.05)$. No difference in the onset of ascites formation was observed between the non-treatment and treatment delay or dose reduction groups in either the HeyA-8 or SKOV3.ip1orthotopic models in this study.

\section{Discussion}

There is minimal information about the impact of treatment delays or dose reductions on treatment responses and overall outcomes. There have been multiple retrospective clinical studies that have clearly demonstrated dose reductions are associated with decrease response rate and overall survival [15-19]. These studies have all evaluated the impact of dose reductions retrospectively and there are always concerns of bias or other variables that may confound results commonly associated with the retrospective study approach. The impact of treatment delays has not been formally evaluated in the clinical setting due to feasibility of creating a homogenous patient population for an un-bias data analysis. However, there is enough retrospective data to support that it would be unethical to attempt to conduct a prospective study to evaluate the impact of chemotherapy treatment delays and dose reductions in human ovarian cancer patients. Hence, the use of human ovarian cancer orthotopic mouse model was ideal to prospectively evaluate the impact of both chemotherapy treatment delays and dose reductions in a controlled setting in a homogenous population. This minimizes impact of outside confounders such as co-morbidities, concomitant medications, previous treatments, differing histology or tumor burden.

In both the HeyA-8 orthotopic model representing the aggressive chemosensitive newly diagnosed ovarian cancer and the SKOV3. iplorthotopic model representing the multi-drug resistant or recurrent ovarian cancer, a decrease in overall survival and ascites formation was observed compared to the full dose on time paclitaxel/carboplatin treatment control group. In addition, the use of growth factors, pegfilgrastim and darbepoetin, significantly improved overall survival and delayed the onset of ascites formation. We hypothesis the addition of growth factors improved the ability of mice to tolerate full dose, on-time chemotherapy which resulted in an improvement in overall survival and tumor response.

This is a prospective study to quantify the impact of dose reductions and treatment delays in an in vivo human ovarian cancer model. This study confirms what in the clinical setting intuitively has been suggested; that both treatment delays and dose reductions have a negative effect on treatment response. This new data can be used to help define the importance of full dose and on time dose of the taxane/platinum agents used in treatment of newly diagnosed and recurrent ovarian cancer. Furthermore, this study demonstrated the benefit of prospective use of growth factors, specifically pegfilgrastim, in combination with the taxane/platinum regimen improved tumor response and ultimately overall survival.

\section{Acknowledgement}

Study was sponsored by unrestricted research grant from Amgen Oncology, INC.

\section{References}

1. Jemal A, Siegel R, Ward E, Murray T, Xu J, Smigal C, Thun MJ (2007) Cancer Statistics, 2007 CA Cancer J Clin 57: 43-66. [Crossref]

2. Bookman MA, Greer BE, Ozols RF (2003) Optimal therapy of advanced ovarian cancer: carboplatin and paclitaxel vs. cisplatin and paclitaxel (GOG158) and an update on GOG 182-ICON5. Int J Gynecol Cancer 13:735-740. [Crossref]

3. Kyrgiou M, Salanti G, Pavlidis N, Paraskevaidis E, Ioannidis JP (2006) Survival benefits with diverse chemotherapy regimens for ovarian cancer: meta-analysis of multiple treatments. J Natl Cancer Inst 98: 1655-1663. [Crossref]

4. Ozols RF, Bundy BN, Greer BE, Fowler JM, Clarke-Pearson D, et al. (2003) Phase III trial of carboplatin and paclitaxel compared with cisplatin and paclitaxel in patients with optimally resected stage III ovarian cancer: a Gynecologic Oncology Group study. J Clin Oncol 21: 3194-3200. [Crossref]

5. Gandhi SK, Arguelles L, Boyer JG (2001) Economic impact of neutropenia and febrile neutropenia in breast cancer: estimates from two national databases. Pharmacotherapy 21: 684-690. [Crossref]

6. Lyman GH, Dale DC, Crawford J (2003) Incidence and predictors of low dose-intensity in adjuvant breast cancer chemotherapy: a nationwide study of community practices. $J$ Clin Oncol 21: 4524-4531. [Crossref]

7. Lyman GH, Kuderer NM, Agboola O, Crawford J, Dale DC (2001) The epidemiology and economics of neutropenia in hospitalized cancer patients: data from the University Healthsystem consortium. Blood 98: 432a.

8. Pettengell R, Gurney H, Radford JA, Deakin DP, James R, et al. (1992) Granulocyte colony-stimulating factor to prevent dose-limiting neutropenia in non-Hodgkin's lymphoma: a randomized controlled trial. Blood 80: 1430-1436. [Crossref]

9. Trillet-Lenoir V, Green J, Manegold C, Von Pawel J, Gatzemeier U, et al (1993) Recombinant granulocyte colony stimulating factor reduces the infectious complications of cytotoxic chemotherapy. Eur J Cancer 29A: 319-324. [Crossref]

10. Ludwig H (2004) rHuEPO and treatment outcomes: the preclinical experience. Oncologist 9 Suppl 5: 48-54. [Crossref]

11. Boogarts M, Mittelman M, Vaupel P (2005) Beyond Anaemia Management: Evolving Role of Erythropoietin Therapy in Neurological Disorders, Multiple Myeloma and Tumour Hypoxia Models. Oncology 69: 22-30. [Crossref]

12. Chandel NS, McClintock DS, Feliciano CE, Wood TM, Melendez JA, et al. (2000) Reactive oxygen species generated at mitochondrial complex III stabilize hypoxiainducible factor-1? during hypoxia. J Biological Chem 275: 25130-25138. [Crossref]

13. Blackwell K, Gascón P, Sigounas G, Jolliffe L (2004) rHuEPO and improved treatment outcomes: potential modes of action. Oncologist 9 Suppl 5: 41-47. [Crossref]

14. Herbert RS, Takeuchi H, Teicher BA (1998) Paclitaxel/carboplatin administration along with antiangiogenic therapy in non-small cell lung and breast carcinoma models. Cancer Chemother Pharmacol 41: 497-504. [Crossref]

15. Mughal TI (2004) Current and future use of hematopoietic growth factors in cancer medicine. Hematol Oncol 22: 121-134. [Crossref]

16. Rosner GL, Hargis JB, Hollis DR, Budman DR, Weiss RB, et al. (1996) Relationship between toxicity and obesity in women receiving adjuvant chemotherapy for breast cancer: results from cancer and leukemia group B study 8541. J Clin Oncol 14: 30003008. [Crossref]

17. Colleoni M, Li S, Gelber RD, Price KN, Coates AS, et al. (2005) Relation between chemotherapy dose, oestrogen receptor expression, and body-mass index. Lancet 366 1108-1110. [Crossref]

18. Epelbaum R, Faraggi D, Ben-Arie Y, Ben-Shahar M, Haim N, et al. (1990) Survival of diffuse large cell lymphoma. A multivariate analysis including dose intensity variables. Cancer 66: 1124-1129. [Crossref]

19. Kwak LW, Halpern J, Olshen RA, Horning SJ (1990) Prognostic significance of actual dose intensity in diffuse large-cell lymphoma: results of a tree-structured survival analysis. J Clin Oncol 8: 963-977. [Crossref]

20. Morrison VA, Picozzi V, Scott S, Pohlman B, Dickman E, et al. (2001) The impact of age on delivered dose intensity and hospitalizations for febrile neutropenia in patients with intermediate-grade non-Hodgkin's lymphoma receiving initial CHOP chemotherapy: a risk factor analysis. Clin Lymphoma 2: 47-56. [Crossref]

Copyright: (C) 2015 Mosley S. This is an open-access article distributed under the terms of the Creative Commons Attribution License, which permits unrestricted use, distribution, and reproduction in any medium, provided the original author and source are credited. 